\title{
Análise dos resultados do uso precoce e tardio da assistência circulatória com balão intra-aórtico (BIA) em pacientes submetidos a correção de cardiopatias com auxílio de circulação extracorpórea
}

\author{
Hugo de Moraes Sarmento MACRUZ*, Oscar Howard FRAZIER ${ }^{\star *}$, Denton A. COOLEY ${ }^{\star \star}$
}

RBCCV 44205-277

\begin{abstract}
MACRUZ, H. M. S.; FRAZIER, O. H.; COOLEY, D. A. - Análise dos resultados do uso precoce e tardio da assistência circulatória com balão intra-aórtico (BID) em pacientes submetidos a correção de cardiopatias com auxílio de circulaçāo extracorpórea. Rev. Bras. Cir. Cardiovasc., 10 (4): 190-197, 1995.
\end{abstract}

RESUMO: Este estudo tem como objetivo, a análise retrospectiva do uso precoce (intra-operatório) e tardio (pós-operatório) do balāo intra-aórtico. Foram estudados 130 pacientes do Texas Heart institute, no período de janeiro a dezembro de 1987 , sendo 103 do sexo masculino e 27 do sexo feminino; a idade média dos pacientes foi de $61,5 \pm 10,76$ (14 a 84) anos, a média de peso foi de $75,5 \pm 16,6$ (42 a 134) kg, a média de superfície corpórea de $1,87 \pm 0,24\left(1,08\right.$ a 2,60) $\mathrm{m}^{2}$ e um tempo médio de circulação extracorpórea de 98 (33 a 299) minutos e de pinçamento aórtico de 49 (10 a 122) minutos. O balão intra-aórtico foi usado em todos os pacientes por baixo débito cardiaco, em 4 associado a disrritmias refratárias a tratamento clínico e 2 por parada cardiorrespiratória, por períodos que variaram de 15 minutos a 256 horas. Os pacientes foram divididos em 6 grupos; Grupol, pacientes que receberam o balāo intra-aórtico no intra-operatório (precoce), Grupo II, pacientes que receberam o balão intra-aórtico no pós-operatório (tardio), Grupo III (pacientes com idade igual ou inferior a 65 anos), Grupo IV (pacientes com idade superior a 65 anos), Grupo V (pacientes com tempo de circulação extracorpórea de até 120 minutos) e Grupo VI (pacientes com tempo de circulação extracorpórea superior a 120 minutos) e 2 subdivisōes nos grupos $\mathrm{V}$ e VI, subgrupo 1/. (pacientes com tempo de pinçamento de aorta menor ou igual a 60 minutos) e subgrupo $2 /$. (pacientes com tempo de pinçamento de aorta maior que 60 minutos). Dos 130 pacientes submetidos ao BIA, $81(62.3 \%)$ sobreviveram e $49(37,7 \%)$ pacientes faleceram; destes, $38(36,2 \%)$ pertenciam ao Grupol e $11(44 \%)$ aoGrupo II. A sobrevida foi maior no Grupo III $(68,6 \%)$ e menor no Grupo IV $(51 \%)$ com p<0,05 mostrando uma diferença estatística com relação à mortalidade no grupo mais idoso. Observamos, também, que, a fração de ejeção, tempo de pinçamento de aorta, não apresentaram diferença estatística significante com relaçāo à mortalidade. Porém a mortalidade com relação ao tempo de CEC foi altamente positiva $(p<0,01)$ para tempo de CEC maior que 120 minutos. Em nossa casuística, o índice de complicaçōes foi de 4,6\% (6 pacientes). Os dados deste estudo sugerem que o balāo intra-aórtico é efetivo como método de suporte circulatório, em pacientes com baixo débito cardiaco pós circulação extracorpórea, havendo tendência a resultados melhores quando usado precocemente; foi observada influência da idade e do tempo de CEC, sugerindo que métodos mais eficientes de assistência circulatória devam ser usados nos pacientes mais idosos e nos pacientes com síndrome de baixo débito cardíaco pós-cirurgia cardíaca com tempos de CEC maiores que 120 minutos.

DESCRITORES: Circulação assistida, balão intra-aórtico. Balão intra-aórtico

Trabalho realizado no Texas Heart Institute, USA.

Recebido para publicaçāo agosto de 1995.

* Fellow do Texas Heart Institute, na época da realizaçăo deste trabalho.

** Do Texas Heart Institute.

Endereço para correspondência: Hugo Macruz. Rua João Moura, 362. CEP: 05412-001 São Paulo, SP, Brasil. Tel.: (011) 289-6730. 
MACRUZ, H. M. S.; FRAZIER, O. H.; COOLEY, D. A. - Análise dos resultados do uso precoce e tardio da assistência circulatória combalão intra-aórtico (BID) em pacientes submetidos a correção de cardiopatias com auxílio de circulação extracorpórea. Rev. Bras. Cir. Cardiovasc., 10 (4):190-197, 1995.

\section{INTRODUÇÃO}

O choque cardiogênico, bem como a síndrome de baixo débito após infarto agudo do miocárdio ou intervenções cirúrgicas com auxílio da circulação extracorpórea (CEC), refratários à terapêutica convencional (drogas vasoativas e inotrópicas) são condições nas quais se tem empregado a assistência circulatória $5,8,20,27,28,30,32$.

O choque cardiogênico ocorre em cerca de $10 \%$ dos pacientes após infarto agudo do miocárdio, e cerca de $1 \%$ a $4 \%$ dos pacientes que são levados à cirurgia com circulação extracorpórea têm um mau prognóstico refletido pela alta mortalidade e morbidade $3,10,25,27$.

O conceito fisiológico da assistência circulatória é o de manter a perfusão tecidual adequada a todos os órgãos, inclusive o coração, sem aumentar as necessidades de energia deste órgão ${ }^{2}$.

Dentre eles, pela disponibilidade, custo, técnica de implante, rapidez e controle, o balão intra-aórtico (BIA) tem tido a preferência da maioria dos centros de cirurgia cardíaca, constituíndo-se em terapêutica auxiliar bem estabelecida $13,17,21$.

Em 1968, KANTROWITZ et alii ${ }^{12}$ relatam a primeira aplicação clínica do BIA em paciente com choque cardiogênico. Desde então, o balão intraaórtico é o sistema mais usado como suporte circulatório 5,13 .

Do exposto, o presente trabalho tem como objetivo a análise do uso do BIA somo método de suporte circulatório, para o tratamento da síndrome de baixo débito cardíaco (SBDC) em pacientes submetidos a correção de cardiopatias com o uso da circulação extracorpórea; o estudo comparativo entre o uso precoce (intra-operatório) e tardio (pósoperatório) do BIA, a análise da mortalidade com relação à idade, ao tempo de perfusão e de pinçamento da aorta e a sua correlação prognóstica com a fração de ejeção pré-operatória, bem como as complicações decorrentes do seu uso.

\section{CASUÍSTICA E MÉTODOS}

Foram estudados, retrospectivamente, $130 \mathrm{pa}$ cientes do Texas Heart Institute, no período de janeiro a dezembro de 1987. A idade média dos pacientes foi de $61,5 \pm 10,76$ (14 a 84 ) anos e uma mediana de 62,5 anos, sendo 27 pacientes do sexo feminino e 103 do sexo masculino (Tabela 1).

O peso médio dos pacientes foi de $75,5 \pm 16,6$ (42 a 134) kg (Tabela 1).

A medida da superfície corpórea (SC) dos pacientes variou de $1,08 \mathrm{~m}^{2}$ a $2,60 \mathrm{~m}^{2}$ com um valor médio de $1,87 \pm 0,24 \mathrm{~m}^{2}$ (Tabela 1$)$.

Dos 130 pacientes estudados, 95 eram portadores de cardiopatia isquêmica, 9 eram portadores de valvopatia crônica de provável etiologia reumática, 3 portadores de aneurisma de ventrículo esquerdo, $1 \mathrm{com}$ aneurisma de aorta, 1 portador de cardiopatia congênita cianótica, 1 pós-transplante cardiaco e 20 eram portadores de cardiopatias associadas (coronariopatia, valvopatia, aneurisma do ventrículo e de aorta) (Tabela 2).

Os 130 pacientes estudados foram submetidos à cirurgia cardíaca com auxílio de circulação extracorpórea. Todos os pacientes apresentavam alteração hemodinâmica caracterizada como choque cardiogênico ou baixo débito cardíaco, durante a saída de CEC, ou na unidade de pós-operatório; apresentavam deterioração do seu estado hemodinâmico, embora estivessem em uso adequado de drogas vasoativas e inotrópicas, sendo, em 4, associado a disritmias cardíacas refratárias e tratamento clínico e, em 2, pós-parada cardiorrespiratória no período de pós-operatório, resultando em um estado hemodinâmico indicativo para o uso do BIA, por períodos que variaram de 15 minutos a 256 horas.

Com o objetivo de avaliar os resultados do emprego do BIA no intra-operatório (período precoce), bem como no pós-operatório (período tardio), e verificar a existência de relação do tratamento usado com a idade dos pacientes, o tempo de CEC, o tempo de pinçamento aórtico, os pacientes foram estudados na seguinte ordem:

TABELA 1

DISTRIBUIÇÃO DOS PACIENTES CONFORME SEXO, IDADE, PESO E SUPERFICIE CORPÓREA

\begin{tabular}{|c|c|c|c|c|c|}
\hline $6 x$ & \multicolumn{2}{|c|}{ SEXO } & \multirow{2}{*}{$\begin{array}{c}\text { IDADE } \\
\text { (anos) } \\
14-84\end{array}$} & \multirow{2}{*}{$\begin{array}{c}\text { PESO } \\
(\mathrm{kg}) \\
42-134\end{array}$} & \multirow{2}{*}{$\begin{array}{c}\begin{array}{c}\text { SUPERFICIE } \\
\text { CORPORAL } \\
\left(\mathrm{m}^{2}\right) \\
1,08-2,60\end{array}\end{array}$} \\
\hline Variaçāo & masc. & fem. & & & \\
\hline Média & 103 & 27 & $61,5 \pm 10,76$ & $75,5 \pm 16,6$ & $1,87 \pm 0,24$ \\
\hline
\end{tabular}


MACRUZ, H. M. S.; FRAZIER, O. H.; COOLEY, D. A. - Análise dos resultados do uso precoce e tardio da assistência circulatória combalão intra-aórtico (BID) em pacientes submetidos a correção de cardiopatias com auxílio de circulação extracorpórea. Rev. Bras. Cir. Cardiovasc., $10(4):$ 190-197, 1995.

TABELA 2

RELAÇĀO DE DISTRIBUIÇÃOO DAS DOENÇAS

\begin{tabular}{lcr}
\hline DOENÇAS & $\begin{array}{c}N^{\circ} \text { DE } \\
\text { PACIENTES }\end{array}$ & $\%$ \\
\hline Cardiopatia isquêmica & 95 & 73,07 \\
Valvopatia crônica & 9 & 6,92 \\
Aneurisma de ventrículo esquerdo & 3 & 2,30 \\
Aneurisma de aorta & 1 & 0,76 \\
Cardiopatia congênita & 1 & 0,76 \\
Transplante cardiaco & 1 & 0,76 \\
Miscelânia & 20 & 15,38 \\
\hline TOTAL & 130 & \\
\hline
\end{tabular}

Grupo I - Pacientes que receberam o BIA no período trans-operatório (105 pacientes).

Grupo II - Pacientes que receberam o BIA no período pós-operatório (25 pacientes).

Grupo III - Pacientes que receberam o BIA e apresentavam idade igual ou inferior a 65 anos (83 pacientes).

Grupo IV - Pacientes que receberam o BIA e apresentavam idade superior a 65 anos (47 pacientes).

Grupo V - Pacientes que receberam o BIA e tiveram um tempo de CEC de até 120 minutos, subdivididos em:

1/. - Pacientes com tempo de pinçamento de aorta menor ou igual a 60 minutos.

2/. - Pacientes com tempo de pinçamento de aorta maior que 60 minutos.

Grupo VI - Pacientes que receberam o BIA e tiveram um tempo de CEC superior a 120 minutos, subdivididos em:

1/. - Pacientes com tempo de pinçamento de aorta igual ou menor que 60 minutos.

2/. - Pacientes com tempo de pinçamento de aorta maior que 60 minutos.
O consolo usado para a geração de energia e controle de insuflação e desinsuflação do balão intraaórtico foi o Datascope, modelo 90 (Datascope Corporation, Paramus, New Jersey, USA).

A análise estatística foi realizada utilizando-se os testes do Qui quadrado e o exato de Fisher.

Finalmente, foram ahalisados: o valor prognóstico da fração de ejeção ventricular esquerda préoperatória, as complicações decorrentes da inserção e uso do BIA.

\section{RESULTADOS}

Dos 130 päcientes submetidos à assistência circulatória com BIA, $81(62,3 \%)$ pacientes sobreviveram e $49(37.7 \%)$ pacientes faleceram (Tabela 3). Dos 49 óbitos ocorridos, $38(77,5 \%)$ ocorreram nas primeiras 24 horas.

TABELA 3

MORTALIDADE GLOBAL

\begin{tabular}{lcc}
\hline PACIENTES & VIVOS & ÓBITOS \\
\hline 130 & $81(62,3 \%)$ & $49(37,7 \%)$ \\
\hline
\end{tabular}

Dos 105 balões intra-aórticos (BIA) instalados nos pacientes do Grupo I (intra-operatório), 67 $(63.8 \%)$ sobreviveram e $38(36.2 \%)$ pacientes faleceram (Tabela 4).

Dos 25 pacientes do Grupo II (pós-operatório), $14(56 \%)$ deles sobreviveram e 11 (44\%) faleceram (Tabela 4). A análise estatística dos resultados de mortalidade dos grupos I e II não apresentou diferença significante.

No Grupo III (pacientes com idade igual ou inferior a 65 anos), 83 pacientes receberam BIA, 57 $(68,6 \%)$ deles sobreviveram e $26(31,3 \%)$ evoluíram para óbito (Tabela 4).

TABELA 4

DISTRIBUIÇÃO DOS PACIENTES E MORTALIDADE NOS GRUPOS I A IV

\begin{tabular}{|c|c|c|c|c|c|c|c|}
\hline \multicolumn{2}{|c|}{$\begin{array}{c}\text { GRUPOI } \\
\text { Intra-operatório }\end{array}$} & \multicolumn{2}{|c|}{$\begin{array}{c}\text { GRUPO II } \\
\text { Pós-Operatório }\end{array}$} & \multicolumn{2}{|c|}{$\begin{array}{l}\text { GRUPO III } \\
\leqslant 65 \text { AnOS }\end{array}$} & \multicolumn{2}{|c|}{$\begin{array}{l}\text { GRUPO IV } \\
>65 \text { AnOS }\end{array}$} \\
\hline $\begin{array}{l}\text { Pac. } \\
105\end{array}$ & $\begin{array}{c}\text { óbitos } \\
38\end{array}$ & $\begin{array}{l}\text { Pac. } \\
25\end{array}$ & $\begin{array}{c}\text { óbitos } \\
11\end{array}$ & $\begin{array}{c}\text { Pac. } \\
83\end{array}$ & $\begin{array}{c}\text { obitos } \\
26\end{array}$ & $\begin{array}{c}\text { Pac. } \\
47\end{array}$ & $\begin{array}{r}\text { óbito } \\
23\end{array}$ \\
\hline$\%$ & $36,2 \%$ & & $44 \%$ & & $31,3 \%$ & & $48,9 \%$ \\
\hline $\begin{array}{c}\text { vivos } \\
67\end{array}$ & $63,8 \%$ & $\begin{array}{c}\text { vivos } \\
14\end{array}$ & $56 \%$ & $\begin{array}{c}\text { vivos } \\
57\end{array}$ & $68,6 \%$ & $\begin{array}{c}\text { vivos } \\
24\end{array}$ & $51,1 \%$ \\
\hline
\end{tabular}


MACRUZ, H. M. S.; FRAZIER, O. H.; COOLEY, D. A. - Análise dos resultados do uso precoce e tardio da assistência circulatória com balão intra-aórtico (BID) em pacientes submetidos a correção de cardiopatias com auxílio de circulação extracorpórea. Rev. Bras. Cir. Cardiovasc., 10 (4):190-197, 1995.

Com relação ao Grupo IV (pacientes com idade superior a 65 anos), 47 pacientes receberam o BIA, dos quais $24(51 \%)$ sobreviveram e $23(48,9 \%)$ faleceram (Tabela 4). A análise estatística comparativa destes 2 grupos, com relação à mortalidade, foi positiva para o grupo acima de 65 anos, com $\mathrm{p}<0,05$.

Grupo V - $101(77,69 \%)$ pacientes (que tiveram um tempo de CEC de até 120 minutos) e subdivididos em;

1/. - Oitenta e três pacientes (até 60 minutos de pinçamento aórtico), tendo $56(67,4 \%)$ pacientes sobrevivido e tendo ocorrido $27(32,5 \%)$ óbitos (Tabela 5).

2/. - Dezoito pacientes (pinçamento aórtico maior que 60 minutos) havendo $13(72,2 \%)$ sobreviventes e ocorrido $5(27,77 \%)$ óbitos (Tabela 5 ).

Grupo VI - com $29(22,30 \%)$ pacientes com tempo de CEC maior que 120 minutos, e subdivididos em;

11. - Dez pacientes (tempo de pinçamento aórtico de até 60 minutos), com $4(40 \%)$ sobreviventes e $6(60 \%)$ óbitos.

21. - Dezenove pacientes (tempo de pinçamento aórtico maior que 60 minutos); $8(42,1 \%)$ sobreviveram e houve $11(57.8 \%)$ óbitos (Tabela 5).

$\mathrm{Na}$ análise estatística dos dois subgrupos, não ficou demonstrada qualquer significância; porém, na análise isolada dos grupos $\mathrm{V}$ e VI, ou seja, pacientes com tempo de CEC menor ou igual e maior que 120 minutos, respectivamente, o resultado estatístico foi altamente signiffcativo para os pacientes do grupo acima de 120 minutos de CEC, com $p<0,01$. Com relação ao tempo de pinçamento aórtico, observamos médias respectivas de 51,65 minutos para os que vieram a falecer e $48,87 \mathrm{mi}$ - nutos, respectivamente, para os sobreviventes, não havendo significado estatístico significativo relativo a evolução desfavorável.

A fração de ejeção como valor prognóstico nos pacientes tratados com BIA não apresentou diferença estatísticamente significante entre 0 grupo de pacientes que vieram a falecer com um valor médio de $38,2 \%$, e o grupo de sobreviventes com um valor médio de $38,6 \%$.

O número total de complicações na casuística apresentada soma $6(4,6 \%)$ pacientes. Isquêmia de membro inferior foi observada em $4(3,07 \%)$ pacientes, $1(0,76 \%)$ paciente apresentou pseudoaneurisma e outro $(0,76 \%)$ laceração arterial, tendo sido tratados cirurgicamente.

\section{COMENTÁRIOS}

Como assinalamos na introdução, a existência da chamada síndrome do baixo débito cardíaco é uma situação na qual existe má perfusão tecidual, que leva a uma hipóxia de graus variados, acarretando a deterioração das funções fisiológicas e que pode, geralmente, culminar com o êxito letal $4,25,27$.

Não raro, pós cirurgia cardíaca com auxílio da CEC, o coração não consegue manter um estado hemodinâmico compatível com a vida, caracterizando a chamada insuficiência cardiaca pós bomba ou baixo débito cardíaco, ou a síndrome pós perfusão.

0 choque cardiogênico e o baixo débito cardíaco após a cirurgia com CEC podem ser conseqüentes a vários fatores, como a hipóxia miocárdica, alterações hidroeletrolíticas e ácido-básicas e, mesmo, a adaptação do miocárdio às modificações estruturais anatômicas conseqüentes à própria cirurgia $15,29,31$.

TABELA 5

DISTRIBUIÇĀO DOS PACIENTES COM RELAÇĀO AO TEMPO DE CIRCULAÇÃO EXTRACORPÓREA, PINÇAMENTO AÓRTICO EÓBITOS

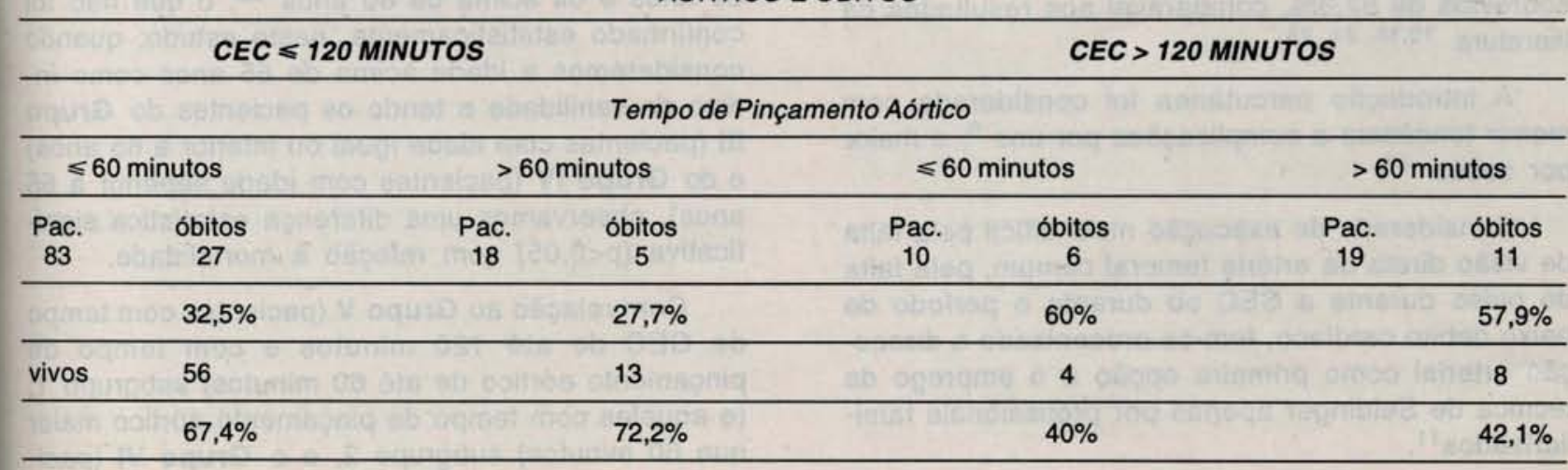


MACRUZ, H. M. S.; FRAZIER, O. H.; COOLEY, D. A. - Análise dos resultados do uso precoce e tardio da assistência circulatória combalāo intra-aórtico (BID) em pacientes submetidos a correção de cardiopatias com auxílio de circulaçāo extracorpórea. Rev. Bras. Cir. Cardiovasc., 10 (4): 190-197, 1995.

As primeiras medidas terapêuticas para o tratamento das alterações conseqũentes a este quadro hemodinâmico são as correções da volêmia, da bioquímica do sangue, do equilíbrio entre a pré e a pós carga, coadjuvado pelo uso de drogas vasoativas e inotrópicas 26 . Entretanto, se, apesar do uso adequado das medidas terapêuticas assinaladas, não houver uma resposta efetiva para a manutenção dos dados pressóricos, do débito cardíaco e da resistência vascular periférica, está indicado o emprego da assistência circulatória mecânica 3,33 .

A assistência circulatória, mais comumente empregada, é a permanência do doente em CEC por períodos variáveis, muitas vezes o suficiente para permitir o retorno a uma adequada função hemodinâmica do coração $18,22,28$. Entretanto, há casos em que só conseguimos uma estabilidade hemodinâmica adequada com a permanência do paciente em CEC. Assim sendo, outras maneiras de auxílio ao coração para melhorar o estado de baixo débito têm sido propostas e, dentre elas, o uso do BIA tem merecido especial atenção pela sua simplicidade de instalação, custo acessível, manuseio relativamente fácil e a possibilidade de seu emprego em hospitais não especializados, bem como permitir uso prolongado e com baixo índice de complicações $5,8,9,13$.

Fisiológicamente, a assistência circulatória deve ser entendida como um método terapêutico que visa manter uma satisfatória perfusão tecidual de todos os órgãos, incluindo o coração, sem aumentar as suas necessidades energéticas ${ }^{2}$.

O BIA aumenta a perfusão coronária, ao aumentar a pressão diastólica na aorta ascendente e diminui o consumo de oxigênio ao reduzir a póscarga $^{9}, 13,16$. Dosagens do lactato no seio coronário comprovam bioquimicamente essa afirmação 13 .

Nos 130 pacientes em baixo débito cardíaco ou choque cardiogênico pós CEC, em nossa casuística, refratários a medidas terapêuticas habituais com drogas vasoativas e inotrópicas, e naqueles nos quais utilizamos o $\mathrm{BIA}$, foi possível obtermos uma sobrevida de $62,3 \%$, comparável aos resultados da literatura $19,10,21,23$

A introdução percutânea foi considerada com menor tendência a complicaçōes por uns ${ }^{5}$, e maior por outros 17 .

Considerada de execução mais difícil pela falta de visão direta da artéria femoral comum, pela falta de pulso durante a CEC ou durante o período de baixo débito cardíaco, tem-se preconizado a dissecção arterial como primeira opção e o emprego da técnica de Seldinger apenas por profissionais familiarizados ${ }^{11}$.
Os dados da literatura demonstram uma incidência de complicações de $2 \%{ }^{8}$, até mais de $36 \% 1,11,13$, a maioria de ordem técnica, como a lesão da artéria puncionada, obrigando à tentativa na artéria contralateral; outros fatores também podem aumentar o insucesso da passagem percutânea do BIA, como a doença arterial periférica prévia, duplicando a possibilidade de complicações, e o sexo feminino ${ }^{13}$. No nosso estudo, complicações como as da literatura foram observadas em $6(4,6 \%)$ pacientes, sendo, em 4 , isquêmia de membro inferior, em 1 pseudoaneurisma e, em outro, laceração arterial durante a retirada do BIA.

$\mathrm{Na}$ nossa casuística foram tentadas punção e inserção percutânea à esquerda, por ser a artéria femoral comum esquerda anatomicamente mais curta e menos tortuosa. Quando não foi possivel a introdução do BIA, optamos pela artéria femoral contralateral ou pela passagem direta pela aorta ascendente. O uso da aorta ascendente denota, muitas vezes, a gravidade do acometimento aterosclerótico, resultando em uma mortalidade alta neste grupo de pacientes ${ }^{33}$. UGORJI et alii 32 referem $71,4 \%$ de mortalidade em pacientes que receberam BIA por via torácica. No grupo estudado, $30(23 \%)$ pacientes receberam BIA via aorta ascendente; $18(60 \%)$ deles faleceram.

Com relação à análise da fração de ejeção préoperatoria, como valor prognóstico, não observamos diferença estatisticamente significante entre a fração de ejeção do ventrículo esquerdo nos sobreviventes $(38,68 \%)$ em relação aos que vieram a falecer $(38,23 \%)$.

No presente estudo os pacientes do Grupo I (pacientes que receberam BIA no período precoce) apresentaram uma mortalidade de $36,2 \%$, e de $44 \%$ no Grupo II (pacientes que receberam BIA no período de pós-operatório); embora os valores sejam diferentes, estatisticamente não apresentaram diferença significante.

Quanto à idade, a literatura demonstra diferentes índices de mortalidades para pacientes de até 60 anos e os acima de 60 anos ${ }^{32}$, o que não foi confirmado estatisticamente, neste estudo; quando consideramos a idade acima de 65 anos como índice de senilidade e tendo os pacientes do Grupo III (pacientes com idade igual ou inferior a 65 anos) e do Grupo IV (pacientes com idade superior a 65 anos), observamos uma diferença estatística significativa $(p<0,05)$ com relação à mortalidade.

Com relação ao Grupo V (pacientes com tempo de CEC de até 120 minutos e com tempo de pinçamento aórtico de até 60 minutos) subgrupo 1 , (e aqueles com tempo de pinçamento aórtico maior que 60 minutos) subgrupo 2, e o Grupo VI (paci- 
MACRUZ, H. M. S.; FRAZIER, O. H.; COOLEY, D. A. - Análise dos resultados do uso precoce e tardio da assistência circulatória com balāo intra-aórtico (BID) em pacientes submetidos a correção de cardiopatias com auxílio de circulação extracorpórea. Rev. Bras. Cir. Cardiovasc., 10 (4):190-197, 1995.

entes com tempo de CEC acima de 120 minutos e com tempo de pinçamento aórtico de até 60 minutos) subgrupo 1 , e (maior que 60 minutos) subgrupo 2, não foi observada significância estatística entre os subgrupos. Na análise comparativa da mortalidade entre os grupos V e VI, (Grupo V, 31,68\% (32/ 101), Grupo VI, $58,62 \%(17 / 29))$, estatísticamente há uma diferença altamente significativa com $p<0,01$. A literatura tem demonstrado que pacientes com longos tempos de CEC e de pinçamento de aorta têm um comprometimento estrutural de tal magnitude que pode haver, como conseqüência, o comprometimento funcional do coração ${ }^{14}$ e que, submetidos ao tratamento com BIA, apresentam meIhor sobrevida ${ }^{8}$.

\section{CONCLUSÕES}

O emprego do BIA em pacientes submetidos a intervenções cirúrgicas com CEC e que apresentam alteração hemodinâmica caracterizada como baixo débito cardíaco nos permite concluir:

- O uso do balão intra-aórtico como método de suporte circulatório, para o tratamento da síndrome de baixo débito cardíaco em pacientes submetidos a correção de cardiopatias com auxílio da CEC na casuística em estudo, mostrou ser um método eficiente, e a análise comparativa entre o uso precoce (Grupo I) e tardio
(Grupo II), não mostrou diferença estatística.

- A análise comparativa em relação à mortalidade dos grupos III e IV é significativa $(p<0,05)$; portanto, para pacientes acima de 65 anos, deverá ser usado outro método de suporte circulatório mais eficiente.

- A análise comparativa entre os grupos V e V com relação à mortalidade e ao tempo de CEC é altamente positiva ( $p<0,01)$, nos alertando sobre os tempos prolongados de CEC.

- A fração de ejeção pré-operatória não demonstrou valor prognóstico no estudo realizado, não havendo contra-indicação ao uso do BIA em pacientes com SBDC e baixa fração de ejeção pré-operatória.

- O número de complicações não contra-indica - uso do BIA, e a sua inserção deverá ser realizada pela técnica percutânea, quando houver pulso evidente e pessoal treinado e, na ausência de pulso, usar a técnica de dissecção.

- A maioria dos óbitos ocorreu nas primeiras 24 horas de pós-operatório, indicando, portanto, que, se o paciente ultrapassa este período, há uma boa possibilidade de recuperação da função cardíaca com BIA, ou então outro método de suporte circulátorio mais eficiente deveria ser utilizado. 
MACRUZ, H. M. S.; FRAZIER, O. H.; COOLEY, D. A. - Análise dos resultados do uso precoce e tardio da assistência circulatória com balão intra-aórtico (BID) em pacientes submetidos a correçāo de cardiopatias com auxílio de circulação extracorpórea. Rev. Bras. Cir. Cardiovasc., 10 (4): 190-197, 1995.

\section{RBCCV 44205-277}

MACRUZ, H. M. S.; FRAZIER, O. H.; COOLEY, D. A. - Analysis of the early and late use of the intra-aortic balloon pump as a method of mechanical support for the management of patients with low output syndrome, following cardiac surgery with cardiopulmonary bypass Rev. Bras. Cir. Cardiovasc., 10 (4): 190-197, 1995.

ABSTRACT: The purpose of this study was to evaluate the intra-aortic balloon pump as a method of mechanical support for the management of patients with low output syndrome, following cardiac surgery with cardiopulmonary bypass. The results with early (intra-operative) and late (post-operative) support were compared, as well as the relationship among cardiopulmonary bypass time, aortic clamp time and mortality. Pre-operative left ventricle ejection fraction, as a prognosis index, and complications rates were also analysed. One hundred and thirty patients from Texas Heart Institute, during the period of January to December 1987 , were studied retrospectively; there were 103 men and 27 women, with a mean age of $61.5 \pm 10.7$ (14 to 84$)$ years. The mean weight body and body surface area were $75.5 \pm 16.6$ (42 to 134) kilograms and $1.87 \pm 0.24$ ( 1.08 to 2.60 ) square meters respectively. The mean time of cardiopulmonary bypass was 98 (33 to 299) minutes and the mean time of aortic clamp was 49 (10 to 122) minutes. All the patients had low cardiac output, associated with refractary arrythmias in 4 and after cardiac arrest in the intensiye care unit in 2 patients. The intra-aortic balloon pump was left for variable periods of time from 15 minutes to 256 hours. The all cohort was divided in six groups, according to the early (intra-operative) insertion of the balloon pump (Group I), the late (post-operative) insertion (Group II), patient's age equal or inferior to 65 years Group III and Group IV age superior to 65 years, Group V and VI according cardiopulmonary bypass time up to 120 or superior to 120 minutes, and both were divided according to aortic clamp time (subgroup 1, up to 60 minutes and subgroup 2 , superior to 60 minutes). The outcome was successful in $81(62.3 \%)$ and $49(37.7 \%)$ died $(36.2 \%$ from Group I and $44 \%$ from Group II). The survival rate was greater in Group III $(68.6 \%)$ than in Group IV (51\%) with p $<0.05$ showing statistical relevance of mortality in the older group. There were no significant associations between ejection fraction, aortic clamp time versus mortality but cardiopulmonary bypass had high statistical significance with $p<0.01$ for times over 120 minutes. Complications were found in 6 patients $(4.6 \%)$. These data suggest that intra-aortic balloon pump is an effective mechanical support for the management of patients with low cardiac output following cardiac surgery with cardiopulmonary bypass, showing a tendency of best results when earliest applied, there is an influence of age and time of cardiopulmonary bypass over the mortality rates, suggesting that in these patients should be used more aggressive and effective circulatory support device.

DESCRIPTORS: Assisted circulation, intra-aortic balloon pump. Intra-aortic balloon pump.

AGRADECIMENTOS: Meus agradecimentos aos Professores Euclydes Fontegno Marques (USP) e Reinaldo Wilson Vieira (UNICAMP), sem os quais este trabalho certamente não existiria.

\section{REFERÊNCIAS BIBLIOGRÁFICAS}

1 BACIEWICZ Jr., F. A.; KAPLAN, B. M.; MURPHY, T. E.; NEIMAN, H. L. - Bilateral renal artery thrombotic occlusion: a unique complication following removal of a transthoracic intra-aortic balloon. Ann. Thorac. Surg., 33: 631-634, 1982.

2 BARBERO-MARCIAL, M. - Circulação assistida. In: ZERBINI, E. J. (ed.) Clínica Círurgica Alipio Corrêa Netto. 3. ed. São Paulo, Sarvier, 1974. p. 436-444.

3 BARBERO-MARCIAL, M. - Síndrome de baixo débito cardíaco. In: STOLF, N. A. G. \& ZERBINI, E. J. Pós-operatório em cirurgia cardiaca. São Paulo, Sarvier, 1979. p. 81-89.

BARBERO-MARCIAL, M.; KAWABE, L.; DALLAN, L. A. O.; RAMIRES, J. A. F.; BELLOTTI, G.;
BITTENCOURT, D.; VERGINELLI, G.; PILEGGI, F ; DECOURT, L. V.; ZERBINI, E. J. - Contrapulsação com balão intra-aórtico em pacientes portadores de infarto agudo do miocardio. Rev. Hosp. Clin. Fac. Med. (São Paulo), 34: 171-176, 1979.
5

6

7
BREGMAN, D. A. - Clinical experience with percutaneous intra-aortic balloon pumping. Cardiovasc. Dis. Bull. Tex. Heart Inst., 7: 318-324, 1980.

6 BREGMAN, D. \& KASKEL, P. - Advances in percutaneous intra-aortic ballon pumping. Critical Care Clin., 2: 221-236, 1986.

7 BREGMAN, D.; PARODI, E. N.; HAUBERT, S. M.; SZARNICKI, R.; EDIE, R. N.; SPOTNITZ, H. M.; BOWMAN, Jr. O. B.; REEMSTMA, K.; MALM, J. R. - Unidirectional intra-aortic balloon pumping and counterpulsation during open heart surgery. In: BREGMAN, D. A. (ed.) Mechanical support of the failing heart and lungs. New York, Appleton-CenturyCrofts, 1977. p. 17-46.

BUCKLEY, M. J. - Intra-aortic balloon circulatory support. In: GRILLO, H. C.; AUSTEN, W. G.; WILKINS, Jr. E. 
MACRUZ, H. M. S.; FRAZIER, O. H.; COOLEY, D. A. - Análise dos resultados do uso precoce e tardio da assistência circulatória com balāo intra-aórtico (BID) em pacientes submetidos a correção de cardiopatias com auxílio de circulação extracorpórea. Rev. Bras. Cir. Cardiovasc., $10(4): 190-197,1995$.

W.; MATHISEN, D. J.; VLAHAKES, G. J. (eds.) Current Therapy in cardiothoracic surgery. Philadelphia, B. C. Decker, 1989, p. 318-319.

9 COSTA, A. R.; TEIXEIRA, G.; PEREIRA, E.; PRATES, P.; KALIL, R. A. K.; LUCHESE, F. A.; SANT'ANNA, J. R.; NESRALLA, I. A. - Emprego do balão intraaórtico em cirurgia cardiaca. Arq. Bras. Cardiol., 59 (Supl. 2): 94, 1992. (Resumo)

10 DILELLO, F. - Results of intraaortic balloon pumping after cardiac sugery: experience with the Percor Balloon catheter. Ann. Thorac. Surg., 46: 442-447, 1988.

11 GOLDMAN, B. S.; HILL, T. J.; ROSENTHAL, G. A.; SCULLY, H. E.; WEISEL, R. D.; BAIRD, R. J. Complications associated with use of the intra-aortic balloon pump. Canadian J. Surg., 25: 153-156, 1982.

12 KANTROWITZ, A.; TJONNELAND, S.; FREED, P. S. Initial clinical experience with intra-aortic balloon pumping in cardogenic shock. J A M A, 203: 113$118,1968$.

13 KERN, M. J. - Intra-aortic balloon counterpulsation. Coron. Artery Dis., 2: 649-660, 1991.

14 KIRKLIN, J. W. \& BARRAT-BOYES, B. G. - Cardiac Surgery. New York. John Wiley \& Sons, 1986. p. 150-166.

15 KOHLER, I.; BOROWSKY, A.; KORB, H.; OZEL, O.; DE VIVE, E. R. - Suporte cardiocirculatorio (SC) em sindrome de baixo débito cardíaco (SBDC) em pósoperatório (PO) de cirurgia cardíaca. Arq. Bras. Cardiol., 61 (Supl. 2): 186, 1993. (Resumo)

16 MARKS, J. D.; KARWANDE, S. V.; RICHENBACHER, W. E.; JONES, K. W.; DOTY, D. B.; MILLAR, R. C.; O'CONNELL, J. B.; RENLUND, D. G.; BRISTOW, M. R.; PENTALOS, G. M. - Perioperative mechanical circulatory support for transplantation. J. Heart Lung Transplant., 11: 117-128, 1992.

17 MARTIN III, R. S.; MONCURE, A. C.; BUCKLEY, M. J.; AUSTEN, W. G.; AKINS, C.; LEIBACK, R. C. Complications of percutaneous intra-aortic balloon insertion. J. Thorac. Cardiovasc. Surg., 85: 186190, 1983.

18 MATTOX, K. L. \& BEALL, A. C. - Application of portable cardiopulmonary bypass to emergency instrumentation. Med. Instrum., 11: 347-349, 1979.

MILLER, R. R. - Combined dopamine and nitroprusside therapy in congestive heart failure. Circulation, 55: 881, 1977. apud BRAUNWALD, E. - Heart Disease. 3. ed. Philadelphia, 1988. Cap. 17, p. 485-543: Management of Heart Failure.

MOULOPOULUS, S. D. - Mechanical assistence in cardiogenic shock. J. Biomater. Appl., 4:544-551, 1989.

MURAD, H.; NASCIMENTO, F. J. V.; NENO, A. C. A.; ALBUQUERQUE, P. C. C.; QUEIROZ, A. D.; FILHO, G. R.; MURAD, M.; SANTOS, M. V. N.; OLIVEIRA, D. A. - Utilização do balão intra-aórtico no pré e pós- operatório de cirurgia cardiaca: experiência do Hospital São Vicente de Paulo - RJ. Arq. Bras. Cardiol., 57: 57, 1991. (Resumo)

NAWA, S.; YAMADA, M.; TERAMOTO, S. - Evaluation of conventional circulatory assist devices: intraaortic balloon pumping, venoarterial bypass, and extracorporeal membrane oxigenation. Chest., 95: 261-266, 1989

PENNINGTON, D. G.; SWARTZ, M.; CODD, J. E.; MERJAVY, J. P.; KAISER, G. C. - Intraaortic balloon pumping in cardiac surgical patients: a nine-year experience. Ann. Thorac. Surg., 36: 125-131, 1983.

PHILLIPS, S. J. - Percutaneous cardiopulmonary bypass and innovations in clinical counterpulsation. Critical Care Clinics, 2: 297-318, 1986.

SCOTT, M. L. \& MILAN, J. R. - Long-term management of intra-aortic balloon pump dependent patient. Angiol. J. Vasc. Dis., March: 192-196, 1983.

SLOGOFF, S. - Dopamine, dobutamine, digitalis, and diuretics during intraaortic balloon support. Cardiovasc. Dis. Bull. Texas Heart Inst., 7: 325-330, 1980.

SMITH, T. W.; BRAUNWALD, E.; KELLY, R. A. Management of heart failure. In: BRAUNWALD, E. (ed.) Heart Disease. 3. ed. Philadelphia, W. B. Saunders, 1988. p. 485.

SPENCER, F. C.; EISEMAN, B.; TRINKLE, J. K.; ROSSI, N. P. - Assisted circulation for cardiac failure following intracardiac surgery with cardiopulmonary bypass. J. Thorac. Cardiovasc. Surg., 49: 56-73, 1965.

SPOTNITZ, H. M.; BERMAN, M. A.; REIS, R. L.; EPSTEIN, S. E. - The effects of synchronized counterpulsation of the pulmonary artery on right ventricular hemodynamics. J. Thorac. Cardiovasc. Surg., 61: 167-170, 1971.

0 STURM, J. T.; FUHRMAN, T. M.; IGO, S. R.; HOLUB, D. A.; McGEE, M. G.; FUQUA, J. M.; NORMAN, J. C. - Quantitative indices of intra-aortic balloon (IABP): dependence during pós-infarction cardiogenic shock. Transac. Am. Soc. Artif. Intern. Organs., 4: 8-12, 1980.

1 TIEFENBRUNN, A. J. - Circulatory support devices in clinical cardiology. Coron. Artery Dis., 2: 637-639, 1991.

UGORJI, C. C.; TURNER, S. A.; McGEE, M. G.; FUHRMAN, T. M.; COOLEY, D. A.; NORMAN, J. C. - Transascending aortic intraaortic balloon insertion with delayed sternal closure: a retrospective analysis. Cardiovasc. Dis. Bull. Texas Heart Inst., 7: 307-315, 1980.

WALDHAUSEN, J. A. \& PIERCE, W. S. - Johnson's Surgery of the chest. 5. ed. Chicago, 1985. p. 269-275. 